\title{
Radiance and Doppler shift distributions across the network of the quiet Sun
}

\author{
H. Tian ${ }^{1,2}$, C.-Y. Tu ${ }^{1,2}$, L.-D. Xia ${ }^{3}$, and J.-S. $\mathrm{He}^{2}$ \\ 1 Max-Planck-Institut für Sonnensystemforschung, Katlenburg-Lindau, Germany \\ e-mail: tianhui924@gmail.com \\ 2 Department of Geophysics, Peking University, Beijing, PR China \\ e-mail: chuanyitu@pku.edu.cn \\ 3 School of Space Science and Physics, Shandong Univ. at Weihai, Weihai, Shandong, PR China \\ e-mail: xld@ustc.edu.cn
}

Received 7 April 2008 / Accepted 27 June 2008

\section{ABSTRACT}

\begin{abstract}
Aims. The radiance and Doppler-shift distributions across the solar network provide observational constraints of two-dimensional modeling of transition-region emission and flows in coronal funnels. These distributions have not, however, been studied in detail and we attempt an investigation for a quiet Sun region.

Methods. Two different methods, dispersion plots and average-profile studies, were applied to investigate these distributions for three EUV lines. In the dispersion plots, we divided the entire quiet Sun region scanned by SUMER into a bright and a dark part according to an image of Fe XII taken by EIT during the scanning; we plotted intensities and Doppler shifts in each bin as determined according to a filtered intensity of Si II. We also studied the difference in height variations of the magnetic field as extrapolated from the MDI magnetogram, in and outside network, in the two parts. For the average-profile study, we selected 74 individual cases and derived the average profiles of intensities and Doppler shifts across the network. Cases with large values of blue shift of Ne VIII were further studied.

Results. The dispersion plots reveal that the intensities of Si II and C IV increase from network boundary to network center in both the bright and dark parts. However, the intensity of Ne VIII shows different trends, namely increasing in the bright part and decreasing in the dark part. In both parts, the Doppler shift of C IV increases steadily from internetwork to network center. The height variations in the magnetic field imply a more homogeneous magnetic structure at greater heights and clearly reflect the different magnetic structures in the two regions. The average-profile study reveals that the intensities of the three lines all decline from the network center to internetwork region. The binned intensities of Si II and Ne VIII have a good correlation. We also find that the large blue shift of Ne VIII does not coincide with large red shift of C IV.

Conclusions. Our results suggest that the network structure is still prominent at the layer where Ne VIII is formed in the quiet Sun, and that the magnetic structures expand more strongly in the dark part than in the bright part of this quiet Sun region. Our results might also hint for a scenario of magnetic reconnection between open funnels and side loops.
\end{abstract}

Key words. Sun: corona - Sun: transition region - Sun: UV radiation - Sun: magnetic fields

\section{Introduction}

Our knowledge of the solar atmosphere, in particular the transition region where temperature increases and density drops dramatically with height, depends largely on spectroscopic observations. Ultraviolet emission lines can provide ample information about the physics of the upper chromosphere, transition region, and lower corona. Since the plasma is optically thin for most emission lines, we can extract information about the physical conditions prevailing in their source regions from the line profiles (see reviews by Mariska 1992 \& Xia 2003).

Before the launch of SOHO (the Solar and Heliospheric Observatory), our understanding of the transition region were largely based on observations made by early spectrographs such as the Naval Research Laboratory (NRL) S082-B EUV spectrograph onboard the Skylab space station (Bartoe et al. 1977), and NRL High-Resolution Telescope Spectrograph (HRTS) flown on some rockets and Spacelab2 (Brueckner et al. 1977; Brueckner \& Bartoe 1983; Brueckner et al. 1986). These early observations provided much valuable information about the solar transition region, which was reviewed by Mariska (1992).
Due to the high spectral and spatial resolution of the SUMER (Solar Ultraviolet Measurements of Emitted Radiation) instrument (Wilhelm et al. 1995; Lemaire et al. 1997) onboard SOHO (Solar and Heliospheric Observatory), many more line profiles were obtained, identified and used in the studies (Curdt et al. 2001, 2004), and the Doppler shifts of these EUV spectral lines can be measured with an accuracy of about $1-2 \mathrm{~km} \mathrm{~s}^{-1}$ (Brekke et al. 1997; Hassler et al. 1999; Peter \& Judge 1999; Wilhelm et al. 2000; Popescu et al. 2004). Besides, another spectrograph CDS (Coronal Diagnostic Spectrometer) onboard SOHO also has the capability of simultaneous multi-wavelength imaging in a number of transition region and coronal lines (Harrison et al. 1995). Thus, our understanding of the solar transition region was improved significantly since 1995 when SOHO was launched.

One of the most prominent features in the chromosphere and transition region is the network structure (Reeves 1976), which is the upward extension of the supergranular boundary at greater heights, and is characterized by clusters of magnetic flux concentrations (Patsourakos et al. 1999). The network manifests itself as bright lanes in the radiance images of chromospheric and transition region lines. The network cell has a size of 
20000-30000 km (Simon \& Leighton 1964), and lasts for 20 to $50 \mathrm{~h}$ (Simon \& Leighton 1964; Schrijver 1997; Raju et al. 1998). The height variation in the network size can be found in Patsourakos et al. (1999), Gontikakis et al. (2003), and Tian et al. (2008b).

Gabriel (1976) proposed the first magnetic network model, in which the transition region emission originates from funnels diverging with height from the underlying supergranular boundary. However, this model failed to reproduce the emission measure at temperatures below $10^{5} \mathrm{~K}$ (Athay 1982). Dowdy et al. (1986) discovered many fine-scale structures of mixed polarities in the photospheric magnetic network; they proposed a modified model in which only a fraction of the network flux opens into the corona as a funnel shape, while the rest of the network is occupied by a population of low-lying loops with lengths less than $10^{4} \mathrm{~km}$. In this model, the small loops are heated internally. Based on SUMER observations, Peter (2001) suggested a new sketch of the structure of the solar atmosphere, which includes different structures that dominate at different heights. The most intriguing feature in this structure is that there are two types of coronal funnels: funnels connected to the solar wind and funnels that are feet of large loops. It might also be possible that the fundamental structure of the solar transition region has not yet been resolved by current observations (Feldman 1983, 1987; Feldman \& Laming 1994; Doschek et al. 2004).

Another prominent characteristic of the solar transition region is the observed redshift of transition region lines (see a review in Mariska 1992). The redshift can reach up to $15 \mathrm{~km} \mathrm{~s}^{-1}$ in the network. In a statistical sense, these redshifts imply the presence of plasma flows or wave motions in the quiet Sun with amplitudes that are substantial fractions of the sound speed (Peter \& Judge 1999). Different mechanisms such as solar wind outflows, spicules, siphon flow through loops, nano-flares, explosive events, and downward propagating MHD waves have been suggested to explain these prominent Doppler shifts of transition region lines (see reviews in Mariska 1992; Brekke et al. 1997; Peter \& Judge 1999; Xia 2003). The redshift is most significant in the middle transition region and decreases to become a blue shift in the upper transition region. The redshift to blueshift transition occurs at electron temperatures of about $5 \times 10^{5} \mathrm{~K}$ and the Doppler shift variation with temperature can be found in Peter \& Judge (1999), Teriaca et al. (1999), and Xia et al. (2004).

There is an increasing consensus that the observed large blue shifts in the upper transition region and lower corona are associated with solar wind outflows in coronal holes. Relative to past studies, a far more accurate rest wavelength of $\mathrm{Ne}$ VIII ( $\lambda 770$, $2 \mathrm{~s}^{2} \mathrm{~S}_{1 / 2}-2 \mathrm{p}^{2} \mathrm{P}_{3 / 2}$ ) which originates in the upper transition region and lower corona, were measured by Dammasch et al. (1999). The Doppler shift of this line can be therefore determined well and has been intensively studied. Sizable areas of large blue shift were found in the Ne VIII dopplergrams in coronal holes and considered to be a signature of solar wind outflow (Hassler et al. 1999; Peter 1999; Stucki et al. 2000; Wilhelm et al. 2000); they tended to be larger in the darker regions of coronal holes (Xia et al. 2003). Hassler et al. (1999) studied the relationship between Doppler shift and chromospheric network, and found that a larger blue shift was closely associated with the underlying chromospheric network. Tu et al. (2005a) inferred that the patches of large Ne VIII blue shift were associated with coronal funnels reconstructed by a force-free field model.

In the quiet Sun, large Ne VIII blue shifts were also found in the network lanes, and considered to be a signature of the solar wind (Hassler et al. 1999) or just mass supply to quiet coronal loops (He et al. 2007; Tian et al. 2008a).
However, the distributions of intensities and Doppler shifts of EUV lines across the network, which are required for 2-D modeling of transition region emissions and flows in coronal funnels, have not been investigated well. Aiouaz et al. (2005b) developed a technique of three-step filter, which was applied to an image of Lymann continuum intensity to reveal the chromospheric network. They binned the filtered continuum intensity and calculated the median value of the Doppler shift of Ne VIII in each intensity bin to produce the so-called dispersion plot. They found that the maximum outflow (blue shift) does not appear in the center of the network but rather near network boundary. Aiouaz (2008) extended this work by studying the redshifts of transition region lines and found the locations of the maximum inflows (redshift) of these lines are network centers in the quiet Sun and network boundaries in the coronal hole. Xia et al. (2004) studied the correlation between radiance and Doppler shift of $\mathrm{Ne}$ VIII and found that a slightly positive correlation exists. They also found that the correlation is not clearly present for the C IV line.

Magnetic field extrapolation is a common way for the solar community to study the magnetic coupling of different solar processes (see e.g. Wiegelmann \& Neukirch 2002; Ruan et al. 2008). With the assumption that the magnetic field may be considered as almost force-free at heights of about $400 \mathrm{~km}$ above the photosphere (Metcalf et al. 1995), Marsch et al. (2004) combined the extrapolated magnetic field based on the force-free model proposed by Seehafer (1978) with EUV observations to study the plasma flows in ARs and detected strong adjacent up and down flows associated with sunspots. A similar method was applied successfully by Wiegelmann et al. (2005), Tu et al. (2005a,c), and Marsch et al. (2006) for a coronal hole study, in Tu et al. (2005b), He et al. (2007), and Tian et al. (2008a) for a quiet sun study, and in Tian et al. (2007) for the study of coronal bright points.

In this paper, we use two different methods, namely the method of dispersion plot and average-profile study, to investigate the distributions of radiance and Doppler shift of three EUV lines across the network in a quiet Sun region.

\section{Observation and data reduction}

On 22 September 1996, a middle-latitude quiet-Sun region was scanned by slit $2\left(1^{\prime \prime} \times 300^{\prime \prime}\right)$ of the SUMER instrument from 00:40 to 08:15 UTC. The raster increment was about $3^{\prime \prime}$. Three strong EUV lines, Si II (153.3 nm), C IV (154.8 nm), and Ne VIII $(77.0 \mathrm{~nm})$ were included in the selected spectral window. This data set was intensively studied before by Dammasch et al. (1999), Hassler et al. (1999), Peter (2000), Gontikakis et al. (2003), Tu et al. (2005b), and Tian et al. (2008a). More details about this observation can be found in these papers. The selected area in our study is $442^{\prime \prime} \times 259^{\prime \prime}$ in size, which is the same as that considered by Tian et al. (2008a).

The standard SUMER procedures for correcting and calibrating the data were applied, including local-gain correction, flat-field correction, geometrical-distortion, and dead-time correction.

Each observed spectrum was fitted by a single Gaussian curve, complemented by a constant and a linear term describing the background. By integrating over a fitted profile, we derived the total count rate for the profile and thus obtained its intensity that was then used to construct the intensity image of each line. The Doppler shift of each line was determined by using the method described by Tu et al. (2005b). 


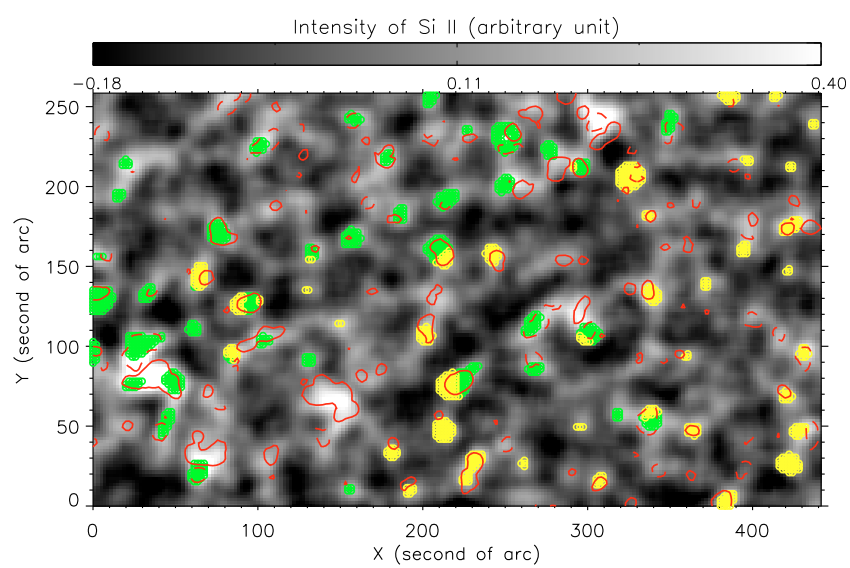

Intensity of $\mathrm{Fe} \mathrm{XII} \mathrm{(arbitrary} \mathrm{unit)}$

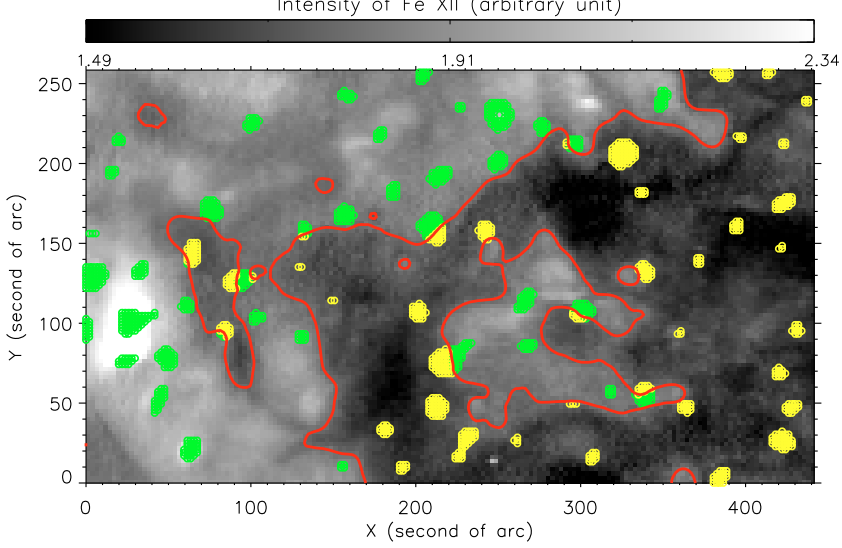

Fig. 1. Upper: the intensity image of Si II, the solid and dashed contours represent strong magnetic fluxes with different polarities (larger than 15 Gauss). Lower: image of EIT-Fe XII observed at 02:30 on 22 September 1996, the red lines mark the boundary between the bright part and the dark part. The intensities are shown in a logarithmic scale. The green and yellow circles mark positions of the data points in bins representing the network center in Figs. 3 and 4, respectively.

We used the magnetogram taken by the Michelson Doppler Imager (MDI) (Scherrer et al. 1995) onboard SOHO on the same day at 01:39 UTC. The pixel size of the magnetogram is about $2^{\prime \prime}$. The correction of the magnetogram and the coalignment of the magnetogram with the SUMER images were carried out by applying the method described by Tu et al. (2005b). The linear Pearson correlation coefficient between the coaligned magnetogram and Si II intensity image is 0.15 (the number of data points is 29436 and the corresponding critical correlation coefficient is 0.01 , so the magnetic field and chromospheric emission correlate very well). The Ne VIII intensity image was used to coalign the SUMER and EIT images. The uncertainties in both coalignments are less than $2^{\prime \prime}$.

We normalized the intensities with respect to the median value over the entire area for each line. The intensity image of $\mathrm{Si}$ II is presented in the upper panel of Fig. 1, where the solid and dashed contours represent strong magnetic fluxes of different polarities (higher than 15 Gauss). The network pattern is clear in this chromospheric line and almost all the strong magnetic fluxes are located in networks. The lower panel of the figure shows the sub-region of a full-disk EIT(Extreme-Ultraviolet Imaging Telescope) (Delaboudinière et al. 1995) image corresponding to the scan area of SUMER. The EIT image was observed in Fe XII at 02:30 on 22 September 1996.

\section{The filtered intensity image of Si ॥}

If the network pattern has a regular shape and the radiance decreases from the center of the network to the internetwork, then the radiance can be used to indicate the distance from the network center. A strong, median, and weak radiance should be found close to the network center, network boundary, and the internetwork region, respectively. In Fig. 1, we can discern that the network structure is very clear and thus the intensity of Si II can be used to represent the distance from the network center.

However, the intensity contrast between the network and internetwork for different supergranular cell is different. To remove the local brightenings and reduce the contrast, we applied a three-step filter technique developed by Aiouaz et al. (2005b) to the intensity image of Si II. The technique was applied instead to an intensity image of Lymann continuum by Aiouaz et al. (2005b). The method can be described in the following way: first we applied a median filter to the image. The width of the median filter was chosen to be $30^{\prime \prime}$, corresponding to the mean size of a supergranular cell. The original intensity image was then divided by the median-filtered image. Finally, the resulting image was smoothed over $10^{\prime \prime}$, which is the usually accepted value of the network size at transition region temperatures (Patsourakos et al. 1999). By using this method, local brightenings and intensity contrasts of network/internetwork for different supergranular cells were reduced although remain in the filtered image. We therefore applied two additional iterations of this method: the method was reapplied to the image produced by the previous filtering iteration. The final filtered intensity image of Si II is presented in Fig. 7.

In this filtered intensity image of Si II, local brightenings have been significantly reduced and the intensity contrasts between the network and internetwork for different supergranular cells are almost comparable. This filtered intensity was used to indicate the distance from network center. A larger, median, and smaller value of the filtered intensity of Si II corresponds to a location in the network center, network boundary, and internetwork region, respectively. Then we are able to complete a statistic study on the trend of a specific parameter from network center to internetwork region, based on this conversion.

\section{Dispersion plots}

Our first method of studying the distributions of radiance and Doppler shift across the network is the method of dispersion plot previously employed by Aiouaz et al. (2005b) and Aiouaz (2008). We binned this filtered intensity image of Si II and calculated the average values of the original intensities and Doppler shifts of the included lines in each bin to produce the dispersion plots.

We divided the entire region into two parts of the same area according to the intensity of EIT-Fe XII. The two parts are labeled as bright part and dark part. The red lines in the lower panel of Fig. 1 indicate the boundary between the bright and dark part. The Fig. 3 of He et al. (2007) illustrated spatial relations between intersection points of the extrapolated open field lines at $4 \mathrm{Mm}$, which implies that the open field lines are located in the dark part, if we compare it with Fig. 1 of this paper. While the bright part is occupied by a population of magnetic loops of different heights (Tian et al. 2008a). Although the correspondence between the open/closed field lines and the dark/bright parts is not exact, a comparison between the statistical study of the two parts should highlight a different property of the two parts. Another reason why we divided the region into two parts 

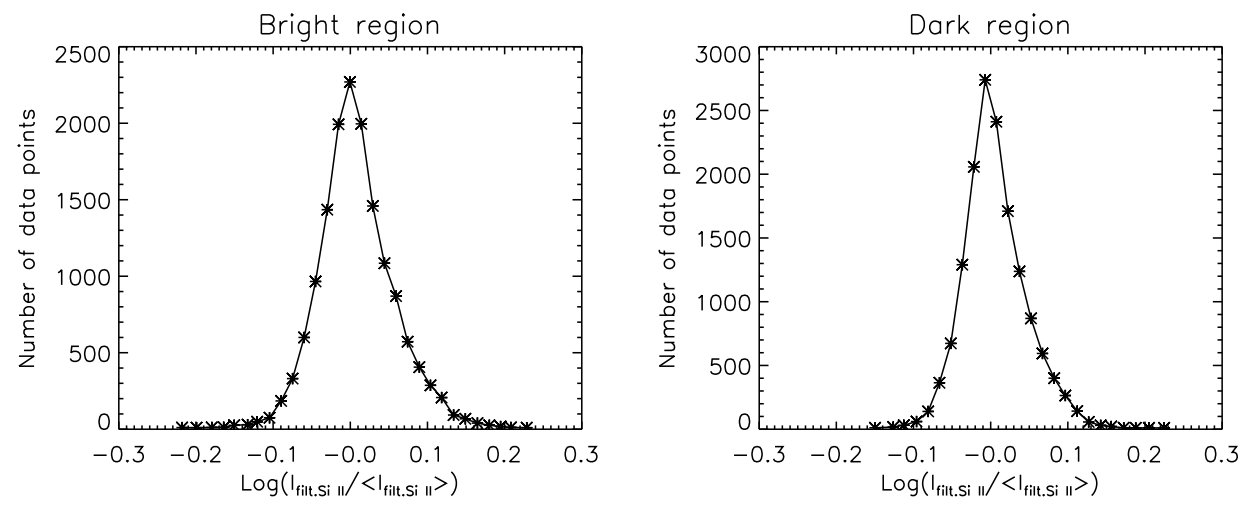

Fig. 2. Histogram of the filtered intensity of Si II in the bright part (left) and the dark part (right).

is that the network emission of Si II in the bright part is systematically higher than that in the dark part. It is therefore more accurate to present dispersion plots for each part separately to reduce the effects of mixing bright internetworks and dark networks.

To study the relationship between the filtered intensity of Si II (an indication of the distance from network center) and the original radiance as well as Doppler shift, we first binned the filtered intensity of $\mathrm{Si}$ II. The number of data points in each bin is shown in Fig. 2. The horizontal axis is given by $\log \left(I_{\text {filt.Si II }} /\left\langle I_{\text {filt.Si II }}\right\rangle\right)$, where $I_{\text {filt.Si II }}$ is the filtered intensity of $\mathrm{Si}$ II and $\left\langle I_{\text {filt.Si II }}\right\rangle$ is the median value of the filtered intensity of Si II over the entire area. It appears that the filtered Si II intensity has a log-normal distribution in both the bright and dark part. The sampling steps of the intensity bins were approximately 0.015 . The minimum number of data points per bin was chosen to be $N_{\min }=10$. If the number of data points in a bin was less than 10 , the step was changed to 0.030 to make a new bin. If the number of data points in the new bin remained less than 10 , we discarded this bin.

We then calculated the average values of the original intensities and Doppler shifts of all three lines and the corresponding standard deviations in each bin. The dispersion plots for the bright and dark part are presented in Figs. 3 and 4, respectively. For each plot, the horizontal axis is the filtered Si II intensity $\left(\log \left(I_{\text {filt.Si II }} /\left\langle I_{\text {filt.Si II }}\right\rangle\right)\right)$, which might be considered to represent the distance from the network center. The higher the filtered Si II intensity, the closer the distance to network center. In each of the two parts, we can determine two thresholds for the filtered Si II intensity by assuming that the network occupies $1 / 3$ of the entire area (Xia et al. 2004) and the network center occupies $1 / 25$ of the network area; these thresholds are indicated by the dashed line representing the outer edge of the network boundary, and the dotted line marking the boundary of the network center in each panel of the figures. In each panel, we can consider that the bins to the left of the dashed line are taken from the internetwork (cell) region, the bins to the right of the dotted line are taken from the network center, and the bins between the dashed line and the dotted line represent the network boundary.

It is unsurprising that the intensities of Si II and C IV show a general increasing trend from network cell to network center, since the network pattern is clearly discernible in intensity images of chromospheric and transition-region lines. This increasing trend is obvious within the network, indicating that network emission increases steadily from the network boundary to network center. The result might suggest that the magnetic fluxes associated with the EUV emission within the network tend to cluster towards the network center. By considering the error bars, the chromospheric and transition-region emission appears, however, to be distributed uniformly in the internetwork region, which suggests that the associated internetwork magnetic fluxes are randomly distributed.

Ne VIII emission originates in the upper transition region and lower corona. In Figs. 3 and 4, we find that the intensity distribution of Ne VIII between internetwork and network center is similar to those of Si II and C IV in the bright part; in the dark part, however, they are not consistent. From the network boundary to the network center, the intensity of Ne VIII shows different trends, increasing in the bright part and decreasing in the dark part. Our previous results demonstrated that magnetic structures expand throughout the upper transition region and lower corona far more significantly with height inside the coronal hole than the quiet Sun (Tian et al. 2008b). And we also found that the dark part is associated with open field lines as extrapolated from the photospheric magnetogram (He et al. 2007; Tian et al. 2008a), which suggests that the dark part has a similar characteristic (expanding stronger than the normal quiet Sun) to that of the coronal hole. Thus, we suggest the decrease of Ne VIII intensity in the network center is due to a more significant expansion of magnetic structures in the dark part.

The Doppler shift of Ne VIII also exhibits a different trends between the network boundary and the network center in the two parts, if we consider only the average value in each bin. In the bright part, the maximum blue shift of Ne VIII is close to the network boundary, while it is found in the network center in the dark part. We note that Aiouaz et al. (2005b) claimed that the blue shift of Ne VIII is strongest at the edge of network patches in a coronal hole, and the adjacent quiet Sun. They conjectured that bipolar fields being swept in from the cell interior cause enhanced magnetic activity with the more or less monopolar network fields and lead to stronger shifts at the network boundary. The result for our bright part data is similar to that of Aiouaz et al. (2005b) and the similar mechanism may also be responsible for it. But in the dark part, where coronal funnels were found (Tian et al. 2008a), the strongest blue shift of Ne VIII appears to be in the center part of the funnel. By using different heating functions, Aiouaz et al. (2005a) developed a two-dimensional MHD model. For one of the heating functions, the maximum blue-shift (outflow) was found not to be in the very center of the funnel but in the vicinity of the center, whereas, for the other heating function, the maximum was aligned well with the center of the funnel. Considering this model, the different locations at which Ne VIII reaches its maximum in the dark and bright part of our data set might be explained as the result of different heating mechanisms dominating in the two parts. However, the significant uncertainties in both our measurements and those of 

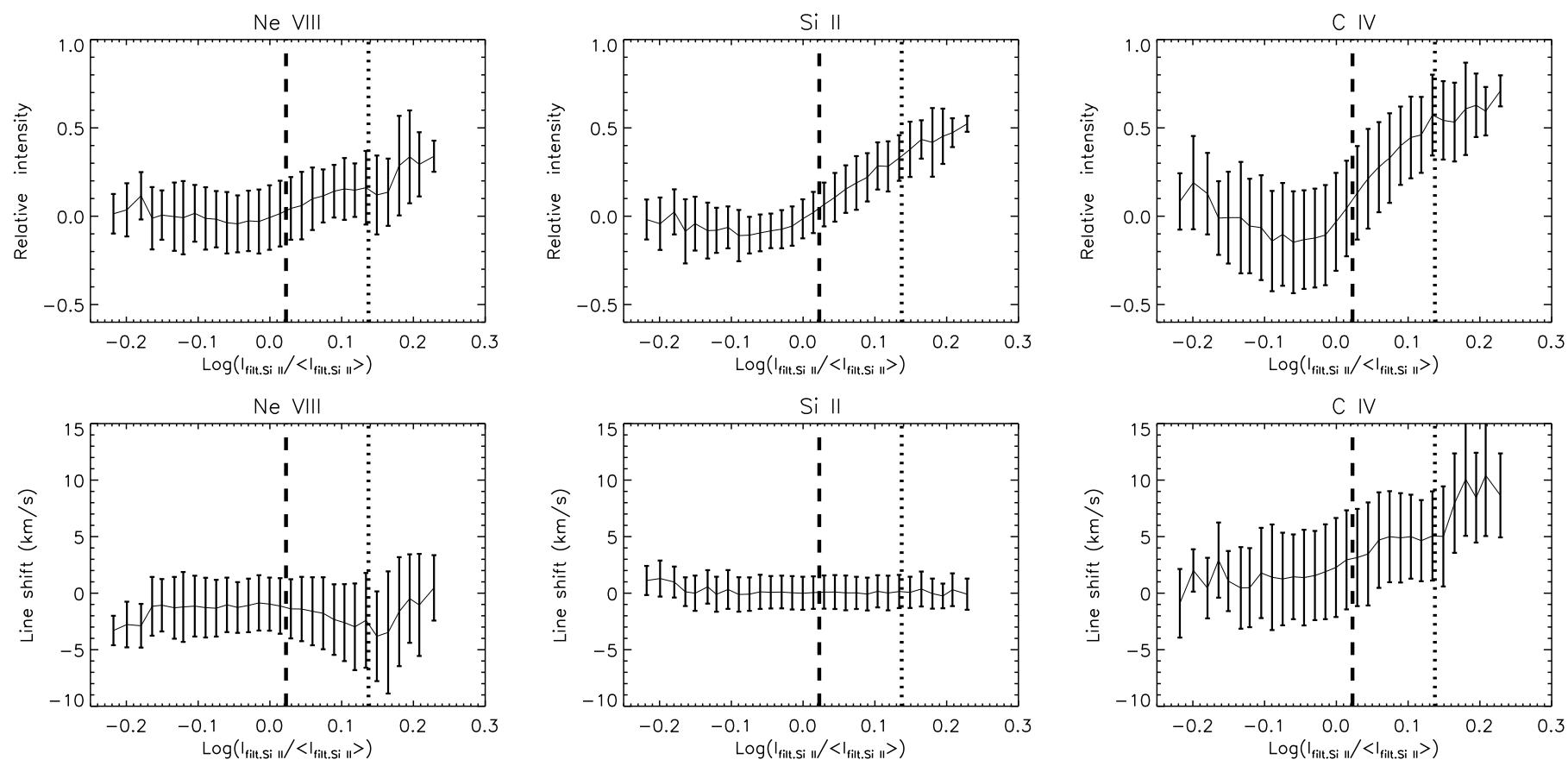

Fig. 3. Dispersion plots for the bright part. The horizontal axis is the filtered Si II intensity $\left(\log \left(I_{\text {filt.Si II }} /\left\langle I_{\text {filt.Si II }}\right\rangle\right)\right)$, which can be considered to represent the distance from network center. A higher $\log \left(I_{\text {filt.Si II }} /\left\langle I_{\text {filt.Si II }}\right\rangle\right)$ represents a position closer to the network center. The dashed line represents the outer edge of network boundary and the dotted line marks boundary of network center.
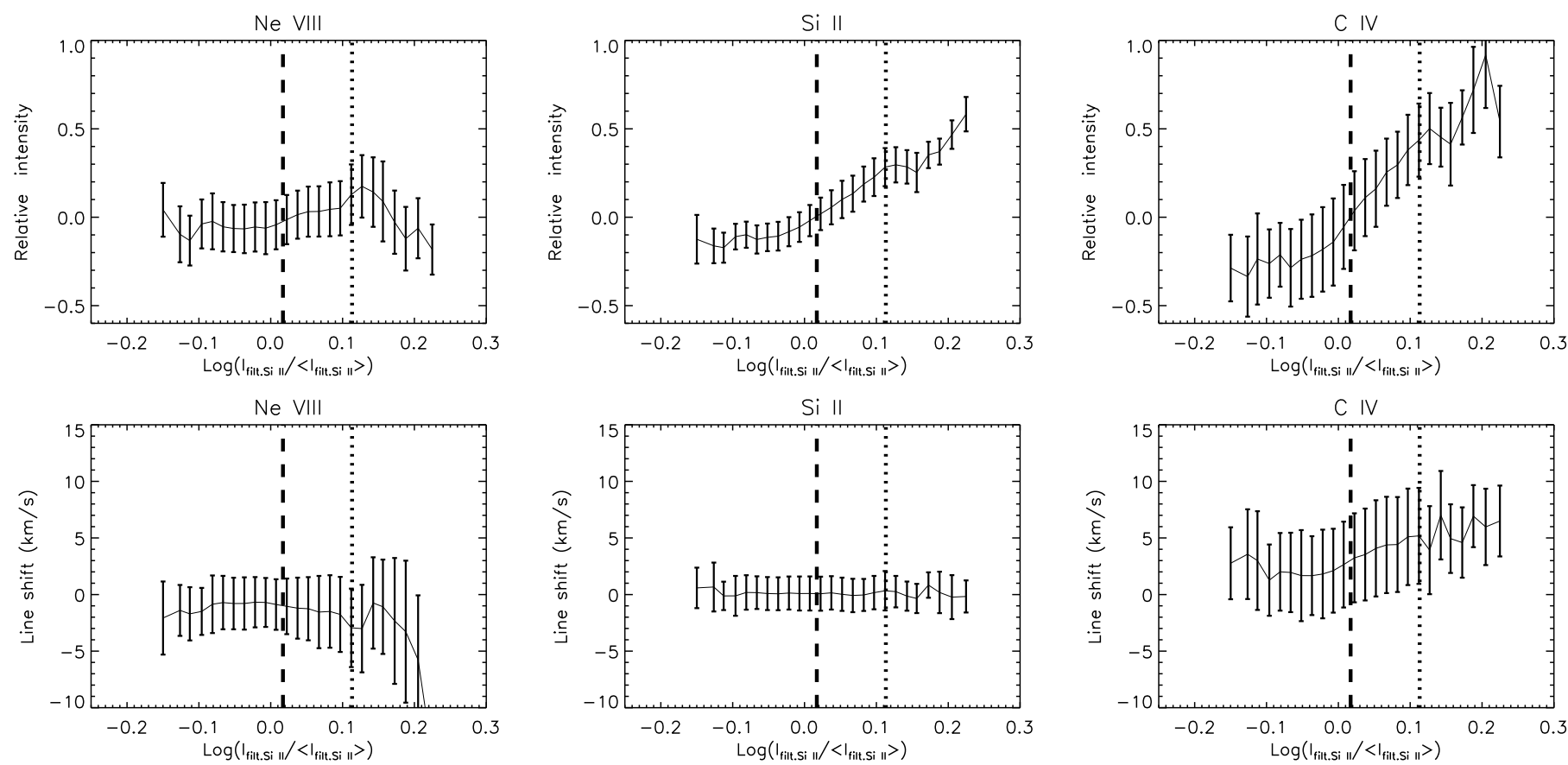

Fig. 4. Dispersion plots for the dark part. The horizontal axis is the filtered Si II intensity $\left(\log \left(I_{\text {filt.Si II }} /\left\langle I_{\text {filt.Si II }}\right\rangle\right)\right)$, which can be considered to represent the distance from the network center. A larger $\log \left(I_{\text {filt.Si II }} /\left\langle I_{\text {filt.Si II }}\right\rangle\right)$ represents a position closer to the network center. The dashed line represents the outer edge of the network boundary and the dotted line marks the boundary of the network center.

Aiouaz et al. (2005b) imply that the above discussion is not very convincing.

Aiouaz (2008) claimed that the maximum inflow (redshift) at transition region temperatures is statistically toward the center of the network in the quiet Sun and toward the boundary of the network in the coronal hole, although large uncertainties were present in their plots. In our case, the redshift of the transition region line $\mathrm{C}$ IV increases steadily from internetwork to network center in both the bright and dark parts, which is similar to the result for the quiet Sun in Aiouaz (2008). The increase is more pronounced in the bright than the dark part.

The Doppler shift of the chromospheric line Si II line remains at the zero level almost everywhere in and outside the network, indicating that the structures of chromospheric loops are 


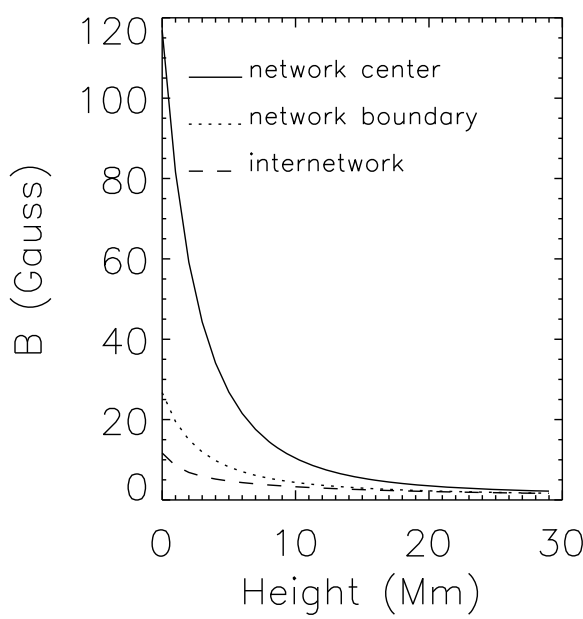

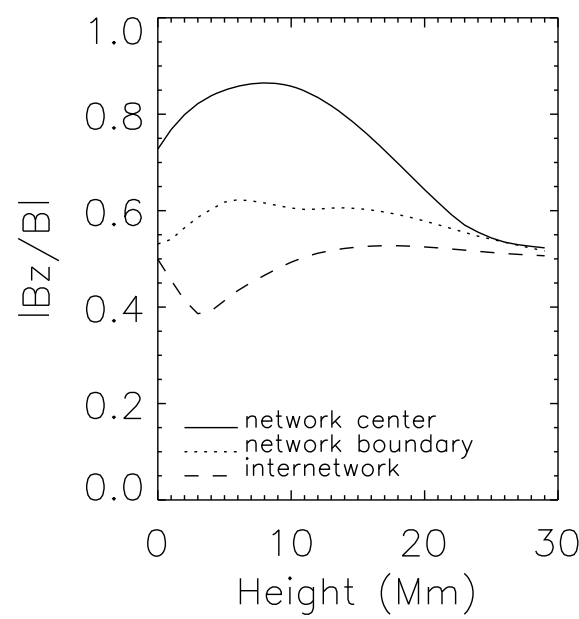

Fig. 5. The height variations in $B$ (left) and $\left|B_{z} / B\right|($ right $)$ for the bright part.

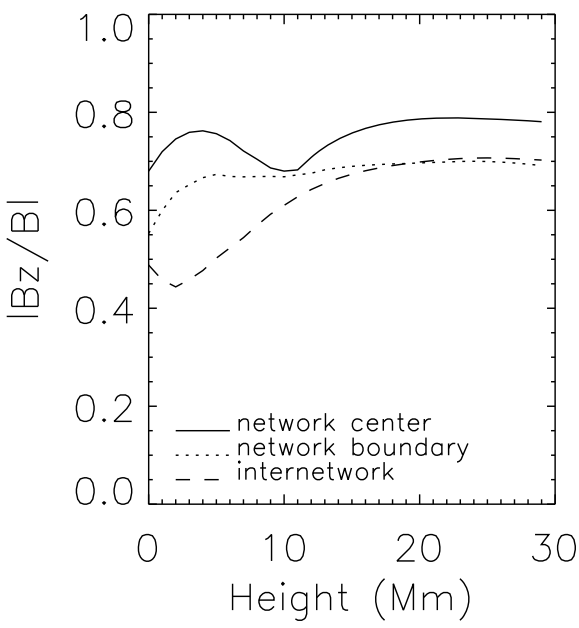

Fig. 6. The height variations in $B$ (left) and $\left|B_{z} / B\right|($ right $)$ for the dark part. similar everywhere in the lower solar atmosphere and there is a balance between upflow and downflow in small chromospheric loops.

At the end of this section, we remind the reader of the principle defect of this dispersion plot method. As stated above, the distance to the network center is represented by, and the bins in Figs. 3 and 4 are divided according to, the filtered intensity of Si II. A comparable intensity contrast between the network and internetwork for different supergranular cells is required of a successful statistical study of the radiance and Doppler shift distributions across the network. Although we applied the threestep filtering method three times to reduce local brightenings, there are still some relatively brighter and darker networks in Fig. 7. In Fig. 1, the green and yellow circles correspond to positions of the data points in bins representing the network center in Figs. 3 and 4, respectively. It is clear that some data points at network boundaries were classified into the network center bins. The results in the network center bins were a mixture of contributions from some network centers and boundaries. In contrast, there are some networks that are insufficiently bright and have a lower filtered intensity of Si II. The intensities and Doppler shifts in the centers of these networks were not considered as contributions to those of the network center bins. This mixture effect is difficult to remove. Applying the three-step filtering method repeatedly can reduce the intensity contrast between the network and internetwork for different supergranular cells. It can also severely destroy the original network pattern. Our choice of three iterations should therefore be appropriate. However, the mixture effect is still existing and should not be neglected in our case. The above discussion might also be the case in Aiouaz et al. (2005b) and Aiouaz (2008).

\section{Height variations of the extrapolated magnetic field}

In our previous works (Tu et al. 2005b; He et al. 2007; Tian et al. 2008a), we extrapolated the magnetic field from the photospheric magnetogram based on a force-free model for this data set. Here we used this previous result but studied the height variations in the extrapolated magnetic field at different locations relative to the network center.

For each of the two parts, we first selected three sets of grid points, which correspond to three bins (one bin at the network center, the other two bins at the network boundary and internetwork) of the filtered Si II intensity, at $0 \mathrm{Mm}$ in our calculation box. We then calculated the average values of the total magnetic field magnitude $B$ and the ratio $\left|B_{z} / B\right|$, where $B_{z}$ is the vertical component of $B$, in each set. After that, we selected three new sets of grid points which have the same $x$ (east-west direction) and $y$ (south-north direction) coordinates as those of the original sets of grid points at different heights in our calculation box. We then calculated the average values of $B$ and $\left|B_{z} / B\right|$ in each new set at differen heights. The height variations in $B$ and $\left|B_{z} / B\right|$ in each set are presented in Fig. 5 for the bright part and Fig. 6 for the dark part. 
The left panels of the two figures clearly show that the magnetic field is far stronger in the network than the network boundary and internetwork at $0 \mathrm{Mm}$, which is a reflection of the wellknown fact that magnetic flux tends to cluster in the network. We can see that the magnetic field strength decreases with height almost exponentially at each of the three selected locations. The difference between the network and internetwork field also decreases with height and reveals a more homogeneous magnetic structure in the upper part of the solar atmosphere.

$\left|B_{z} / B\right|$ is an indicator of the inclination of a magnetic field line with respect to the horizontal direction. The right panels of the two figures reveal that $\left|B_{z} / B\right|$ is much larger inside the network than at the network boundary and internetwork region at lower heights above the photosphere. This is easy to understand because there are more open field lines and funnel structures originating in the network than the internetwork. Above $10 \mathrm{Mm}$, the difference in $\left|B_{z} / B\right|$ between the three locations appears, however, to decrease with height. In the bright part, they are equal to each other at about $30 \mathrm{Mm}$, where the value of $\left|B_{z} / B\right|$ is about 0.5 . This result suggests a more homogeneous magnetic structure dominated by large loops with all possible inclinations in the upper part of the solar atmosphere (Peter 2001). We found that the values of $\left|B_{z} / B\right|$ at $30 \mathrm{Mm}$ are far larger (about $0.7-0.8$ ) than 0.5 in the dark part, which is consistent with our previous result that there are open field lines (He et al. 2007; Tian et al. $2008 \mathrm{a}$ ) in this part. We note that the value of $\left|B_{z} / B\right|$ at $30 \mathrm{Mm}$ above the network center is larger than values at the other two selected locations. This result may be explained that open field lines tend to stretch up vertically from the network centers.

\section{Average-profile study}

The second method used in our study of the distributions of radiance and Doppler shift across the network is an average-profile study. This method aims to study the average trend of radiance and Doppler shift from internetwork region to network by selecting some individual cases.

We chose 74 parallelogram regions, each of which stretches from one network cell to a neighboring cell across the bridging network; in our case they are close to the places where more than two network lanes intersect. In Fig. 7, the selected cases are outlined in black in the filtered intensity image of Si II. In both panels, the red network lines are taken from Hassler et al. (1999) and can be used as a good approximate of the network pattern.

For each case, we first averaged the value of $\log \left(I_{\text {filt.Si II }} /\left\langle I_{\text {filt.Si II }}\right\rangle\right)$ along the short side of the parallelogram region and obtained the distribution of $\log \left(I_{\text {filt.Si II }} /\left\langle I_{\text {filt.Si II }}\right\rangle\right)$ along the long side. We found the position of the maximum value of $\log \left(I_{\text {filt.Si II }} /\left\langle I_{\text {filt.Si II }}\right\rangle\right)$ in the parallelogram region and defined this to be the network center, we then defined the two locations where $\log \left(I_{\text {filt.Si II }} /\left\langle I_{\text {filt.Si II }}\right\rangle\right)$ declines to $92 \%$ of its maximum value to be the network boundaries (still in the parallelogram region) on both sides of the network center. By using this definition we can calculate the average size of the 74 networks. We obtained the value of $9.88 \pm 0.29$ arcsec, which is consistent with the result of Patsourakos et al. (1999).

We then defined the network center to be the position 0 , and the two network boundaries to be position 1 . We extended the range $0-1$ twice on both sides. The new boundaries were defined as 2 . We divided the range $0-2$ into 10 bins according to the position on each side, that is $0-0.2,0.2-0.4,0.4-0.6,0.6-$ $0.8,0.8-1.0,1.0-1.2,1.2-1.4,1.4-1.6,1.6-1.8,1.8-2$, which we referred to as the normalized distance from the network center. Then the two bins on different sides of the network center

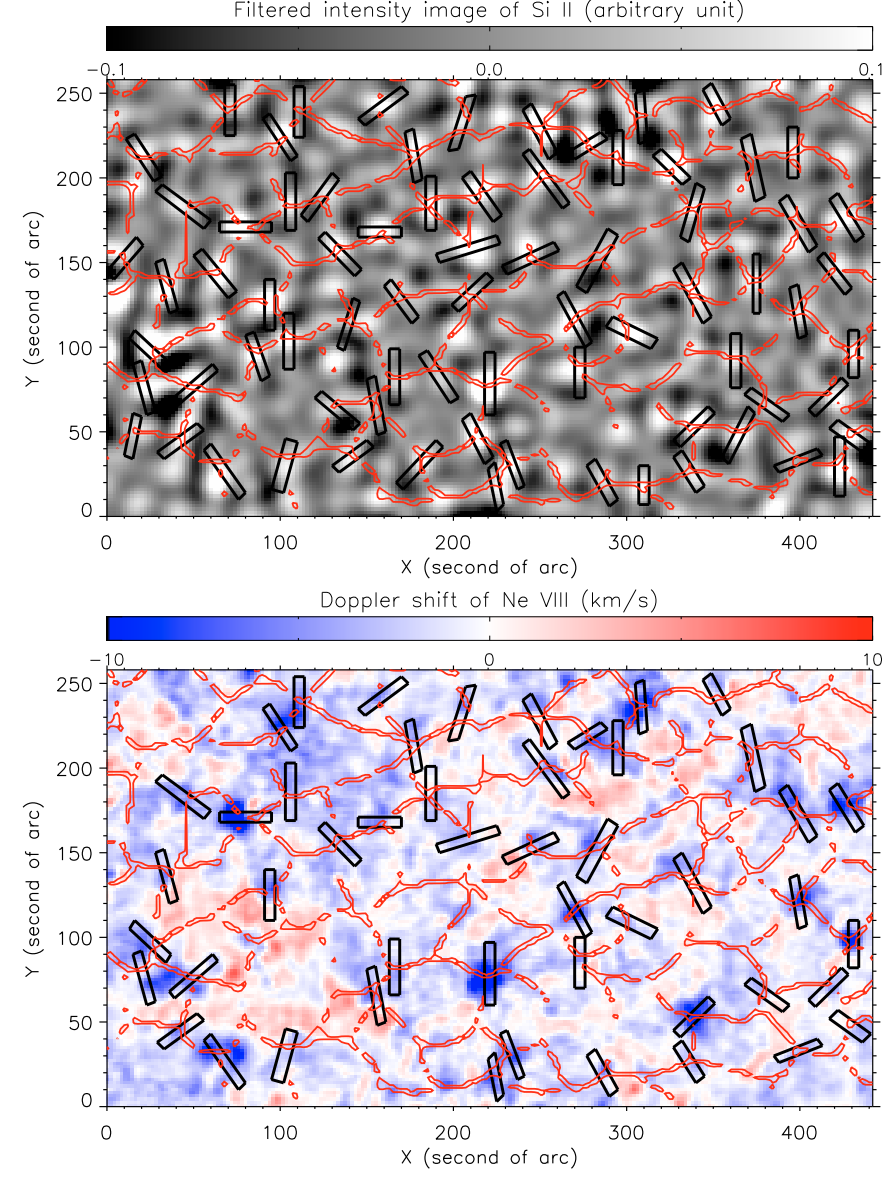

Fig. 7. Upper: the filtered intensity image of Si II. The selected cases are outlined in black. Bottom: the Dopplergram of Ne VIII. The largeblueshift cases are outlined in black. In both panels, the red network lines are taken from Hassler et al. (1999).

with the same normalized distance from the network center are combined into one bin. Therefore, there were only 10 bins or each case and we calculated the average values of intensity and Doppler shift for every line in each bin.

We applied the method mentioned above to each case and obtained the profiles of the intensity and Doppler shift of every line across the network. We then normalized the intensity and Doppler shift in the form of $\frac{A-A_{\min }}{A_{\max }-A_{\min }}$, where $A$ is the value of line parameter (intensity or Doppler shift), and $A_{\min }$ and $A_{\max }$ are the minimum and maximum value of the parameter in the normalized distance range $0-2$. We named this quantity the normalized difference of intensity or shift. For the Doppler shift of Ne VIII, $A$ is used as its minus value in order to study the blue shift of this line. We then obtained the normalized profiles of the line parameters across the network for each case. For all 74 cases, we extracted the 74 normalized values of each parameter in the bin at the same normalized distance, and calculated the mean value and the associated standard deviation. Then we obtained an average normalized profile of each line parameter, which is shown in Fig. 8.

General trends of the radiance and Doppler shift distributions across the network are revealed in Fig. 8. We found that the emissions of the three lines all declines from the network center to internetwork region. The binned intensities of Si II and C IV decrease slowly from network center to network boundary and more significantly in the internetwork region. They have a correlation coefficient of 0.99 . The correlation coefficient between the 

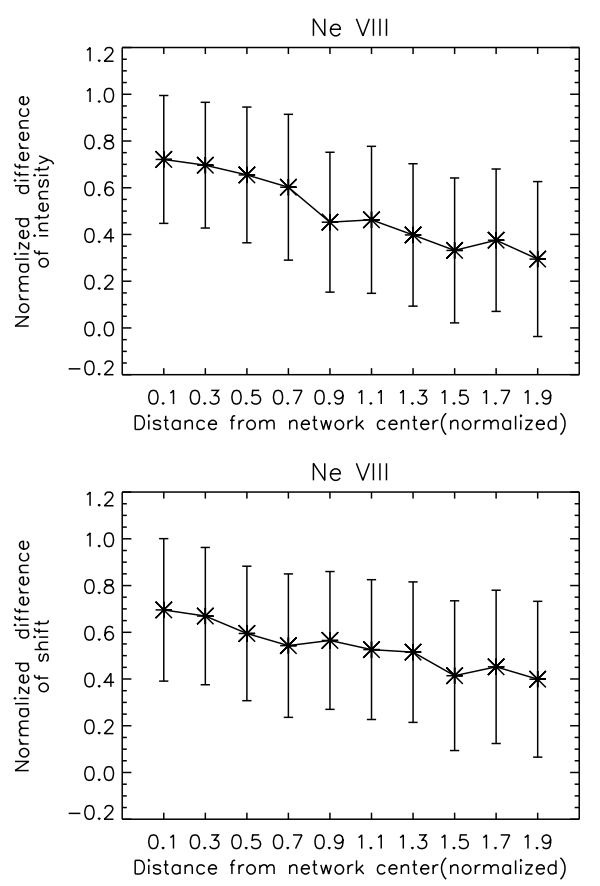

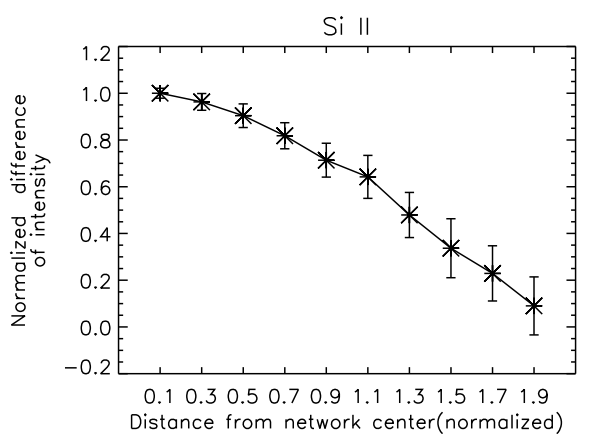

Si II

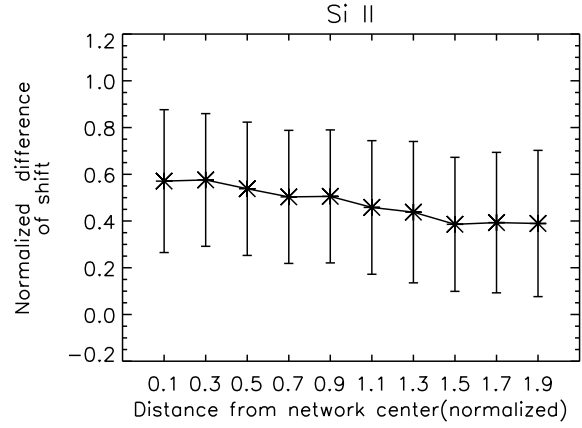

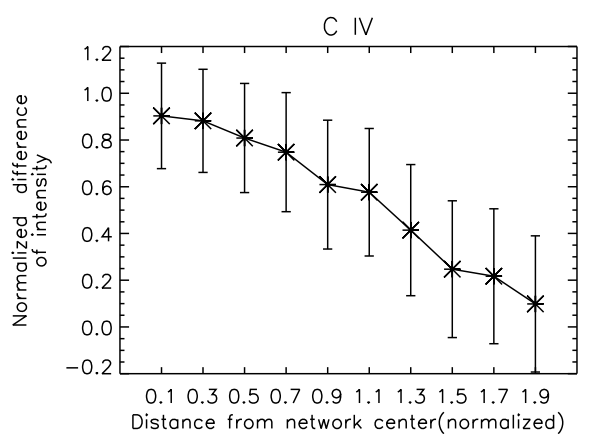

C IV

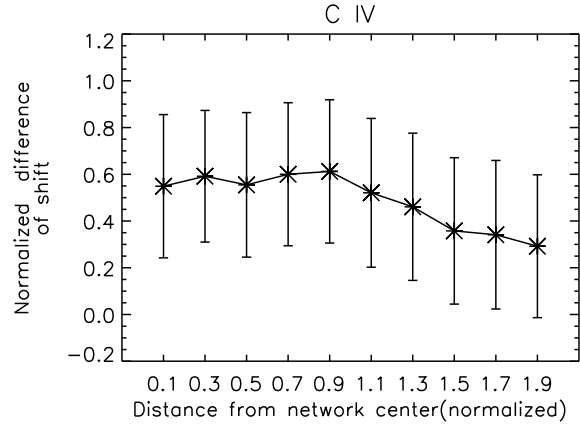

Fig. 8. Average profiles of radiance and Doppler shift for the selected cases.

binned intensities of Si II and Ne VIII is 0.95 , indicating that the network structure is still prominent in the upper transition region of the quiet Sun.

Although with large uncertainty, the general trend of the $\mathrm{Ne}$ VIII blue shift decreases from the network center to the internetwork region. The redshift of C IV is higher in the network and continues to decrease beyond network boundary. As for Si II, its Doppler shift appears to be slightly higher in the network than the internetwork, which may be due to the stronger magnetic activity in the network. These Doppler-shift curves can provide a measure of only the average trend and we are unable to draw firm conclusions because of the significant size of the uncertainties.

\section{Result of large-blueshift cases}

It has been found that large blue patches seen in the dopplergram of Ne VIII are associated with the chromospheric network (Hassler et al. 1999). Since a large blue shift of Ne VIII is considered to be a signature of the solar wind (Hassler et al. 1999; Tu et al. 2005a) or mass supply to quiet coronal loops (He et al. 2007; Tian et al. 2008a), it would be interesting to study the distribution of the Ne VIII blue shift in those network regions around where large blue shift of Ne VIII is found.

We selected cases for which the maximum Ne VIII blue shift is higher than $5 \mathrm{~km} \mathrm{~s}^{-1}$ in the normalized distance range $0-2$, from the 74 cases described in the previous section. We show 49 cases in the bottom panel of Fig. 7, for which our criterion is met. The mean size of the network is $9.05 \pm 0.53 \operatorname{arcsec}$ for these large-blueshift cases. It is slightly smaller than the average network size of all cases. We then constructed in a similar way an average normalized profile of each line parameters. The results are presented in Fig. 9.

The contrast between the intensity in and outside the network is significant for Si II and C IV, which is natural and should be the case for almost every supergranular cell. However, the intensity profile for Ne VIII is rather flat, which suggests that the magnetic funnels, whether being the footpoints of large loops (Peter 2001) or associated with the solar wind (Tu et al. 2005a), expand and stretch into the region above the internetwork at the heights where considerable outflows of Ne VIII are found.

As for the Doppler shift, we found that the highest blue shift of Ne VIII does not coincide with the highest redshift of C IV. The blue shift of Ne VIII decreases steadily from the network center to the internetwork region, while the redshift of C IV reaches its maximum close to the network boundary (the normalized distance 0.7-1.1). This result appears to be consistent with the scenario proposed by Axford et al. (1999) and Tu et al. (2005c), in which reconnection occurs between the open funnels and side loops. The outflows produced by reconnections around a funnel tend to converge towards the center of the funnel. In contrast, the hot plasma trapped in low-lying loops are pulled down when they cool, and the downflows are stronger at the boundary of the network where side loops are accumulated. The Doppler shift of Si II appears to be slightly higher in the network than in the internetwork region. We propose that it is due to the stronger magnetic activity in the network, although the structures of chromospheric loops are similar in and outside the network.

The detailed distributions of radiance and Doppler shift across the network at different layers (chromosphere, transition region, and corona) above the photosphere have not been studied systematically. Our work with that of Aiouaz et al. (2005b) and Aiouaz (2008) may be a first step in analyzing these distributions. The 1-D modeling of the acceleration of the solar wind has been investigated intensively. With the improvement of current observations, it is increasingly important to develop 2-D and 3-D models to study the origin and acceleration of the solar wind. We believe that the above observation results are helpful for 2-D modeling of network emission and outflows in coronal funnels.

\section{Summary}

We have completed a statistical analysis of the distributions of both the radiance and Doppler shift across the network at different layers (chromosphere, transition region, and lower corona) above the photosphere. We applied the filtering technique 


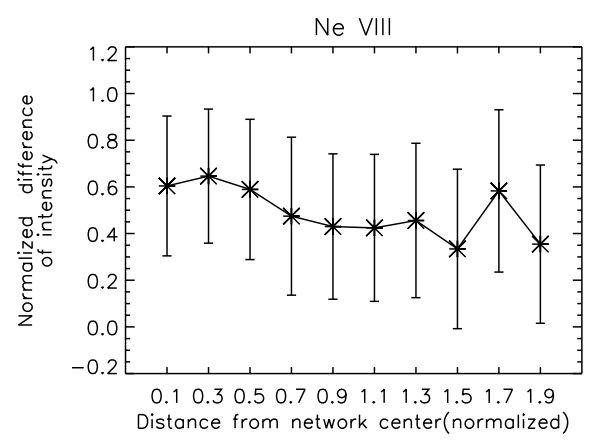

$\mathrm{Ne}$ VIII

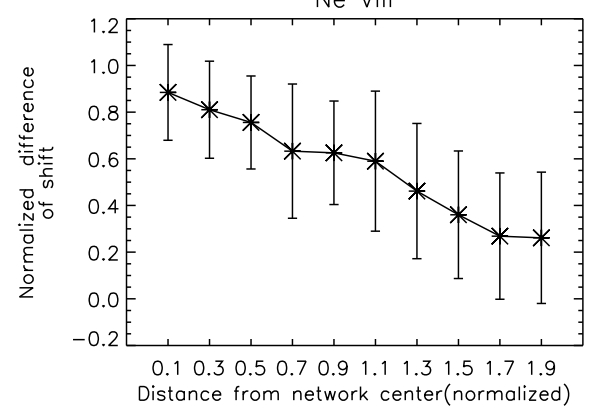

Si II

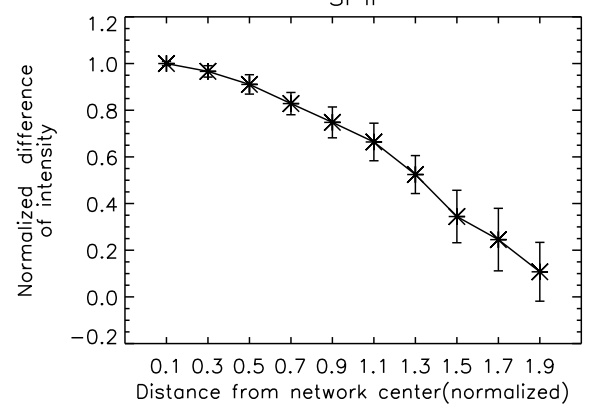

Si II

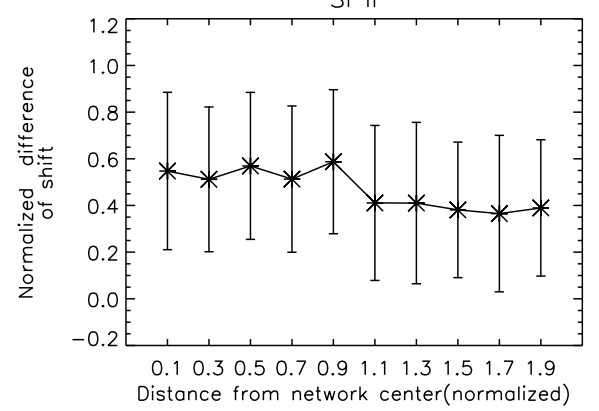

C IV

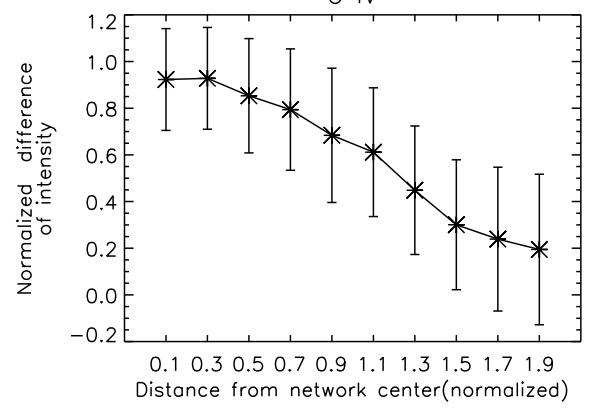

C IV

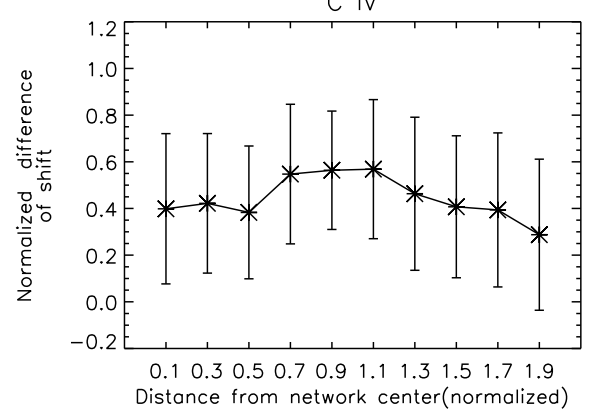

Fig. 9. Average profiles of radiance and Doppler shift for the large-blueshift cases.

developed by Aiouaz et al. (2005b) to an intensity image of the chromospheric line Si II obtained by SUMER/SOHO in a quiet Sun region. The filtered intensity image of Si II was used to reveal the network. Two different methods, namely the method of dispersion plot and the average-profile study, were used to investigate the distributions of radiance and Doppler shift of Si II, $\mathrm{C}$ IV, and Ne VIII across the network.

We divided the entire quiet Sun region into a bright and a dark part, and produced dispersion plots for each part. The intensities of Si II and C IV show an obvious increasing trend from network boundary to network center in both parts. However, the intensity of Ne VIII shows different trends, namely increasing in the bright part and decreasing in the dark part. This result might suggest a stronger expansion of magnetic structures in the dark part than in the bright part. In each of the two parts, the Doppler shift of C IV increases almost steadily from internetwork to network center. The average Doppler shift of the chromospheric line Si II remains at the zero level in and outside network. The distribution of Ne VIII Doppler shift is not clear due to the large uncertainties. This method largely depends on the intensity contrasts between the network and internetwork for different supergranular cells. The mixture effect influences the statistical results and could not be neglected because it is difficult to obtain a comparable contrast for different supergranular cells in real conditions.

The difference between height variations of the extrapolated magnetic field above the internetwork, network boundary, and network center was also investigated; this difference implies a more homogenious magnetic structure at greater height and clearly reflects the different magnetic structures in the two parts.

By selecting individual cases and investigating the average profiles of radiance and Doppler shift distributions across network, we found that the intensities of the three lines all decline from the network center to internetwork region. The correlation coefficient between the binned intensities of Si II and Ne VIII is 0.95 , indicating that the network structure is still prominent at the layer where Ne VIII forms in the quiet Sun. Although with large uncertainties, the average Ne VIII blue shift decreases from the network center to internetwork region. The redshift of C IV is higher in the network and decreases beyond the network boundary.

Since the high blue shift of Ne VIII could be a signature of solar wind or mass supply to coronal loops, we also studied the average profiles of radiance and Doppler shift across network for the large-blueshift cases. The intensity distribution of Ne VIII does not correlate with those of Si II and C IV. We found that the blue shift of Ne VIII decreases steadily from the network center to the internetwork region, and the large blue shift of Ne VIII does not coincide with large red shift of C IV.

Our observation results are worthy of consideration in terms of 2-D modeling of network emissions and outflows in coronal funnels.

Acknowledgements. The SUMER project is financially supported by DLR, CNES, NASA, and the ESA PRODEX programme (Swiss contribution). SUMER, MDI, and EIT are instruments on board SOHO, an ESA and NASA mission. We thank the teams of SUMER, MDI, and EIT for using their data. We also thank the referee for his careful reading of the paper and for the comments and suggestions.

H. Tian, C.-Y. Tu, and J.-S. He are supported by the National Natural Science Foundation of China (NSFC) under contracts 40574078 and 40436015. H. Tian is now supported by China Scholarship Council for his stay in the Max-PlanckInstitut für Sonnensystemforschung in Germany. L.-D. Xia is supported by NSFC under Grant 40574064 and the Programme for New Century Excellent Talents in University (NCET).

\section{References}

Athay, R. G. 1982, ApJ, 263, 982

Aiouaz, T. 2008, ApJ, 674, 1144

Aiouaz, T., Peter, H., \& Keppens, R. 2005a, A\&A, 442, L35

Aiouaz, T., Peter, H., \& Lemaire, P. 2005b, A\&A, 435, 713

Axford, W. I., Mckenzie, J. F., Sukhorukova, G. V., et al. 1999, Space Sci. Rev., 87,25

Bartoe, J.-D. F., Brueckner, G. E., Purcell, J. D., \& Tousey, R. 1977, Appl. Opt., 16,879

Berger, T. E., \& Lites, B. W. 2003, Sol. Phys., 213, 213

Brekke, P., Hassler, D. M., \& Wilhelm, K. 1997, Sol. Phys., 175, 349

Brueckner, G. E., \& Bartoe, J.-D. F. 1983, ApJ, 272, 329

Brueckner, G. E., Bartoe, J.-D. F., \& VanHoosier, M. E. 1977, in Proc. OSO-8, Workshop, ed. E. Hansen, \& S. Scha.ner (Boulder: Univ. Colorado Press), 380 
Brueckner, G. E., Bartoe, J.-D. F., Cook, J. W., Dere, K. P., \& Socker, D. G. 1986, Adv. Space Res., 6, 263

Chae, J., Schühle, U., \& Lemaire, P. 1998, ApJ, 505, 957

Curdt, W., Brekke, P., Feldman, U., et al. 2001, A\&A, 375, 591

Curdt, W., Landi, E., \& Feldman, U. 2004, A\&A, 427, 1045

Dammasch, I. E., Wilhelm, K., Curdt, W., \& Hassler, D. M. 1999, A\&A, 346, 285

Delaboudinière, J.-P., Artzner, G. E., \& Brunaud, J. 1995, Sol. Phys., 162, 291

Doschek, G. A., Mariska, J. T., \& Akiyama, S. 2004, ApJ, 609, 1153

Dowdy, J. F., Jr., Rabin, D., \& Moore, R. L. 1986, Sol. Phys., 105, 35

Feldman, U. 1983, ApJ, 275, 367

Feldman, U. 1987, ApJ, 320, 426

Feldman, U., \& Laming, J. M. 1994, ApJ, 434, 370

Gabriel, A. H. 1976, Philos. Trans. R. Soc. London A, 281, 575

Gontikakis, C., Peter, H., \& Dara, H. C. 2003, A\&A, 408, 743

Harrison, R. A., Sawyer, E. C., Carter, M. K., et al. 1995, Sol. Phys., 162, 233

Hassler, D. M., Dammasch, I. E., Lemaire, P., et al. 1999, Science, 283, 810

He, J.-S., Tu, C.-Y., \& Marsch, E. 2007, A\&A, 468, 307

Lemaire, P., Wilhelm, K., Curdt, W., et al. 1997, Sol. Phys., 170, 105

Mariska, J. T. 1992, The Solar Transition Region (Cambridge: Cambridge Univ. Press)

Marsch, E., Wiegelmann, T., \& Xia, L.-D. 2004, A\&A, 428, 629

Marsch, E., Zhou, G.-Q., He, J.-S., \& Tu, C.-Y. 2006, A\&A, 457, 699

Metcalf, T. R., Jiao, L., McClymont, A. N., \& Canfield, R. C. 1995, ApJ, 439, 474

Peter, H. 1999, ApJ, 516, 490

Peter, H. 2000, A\&A, 360, 761

Peter, H. 2001, A\&A, 374, 1108

Peter, H., \& Judge, P. G. 1999, ApJ, 522, 1148
Patsourakos, S., Vial, J.-C., Gabriel, A. H., \& Bellamine, N. 1999, ApJ, 522, 540 Popescu, M. D., Doyle, J. G., \& Xia, L. D. 2004, A\&A, 421, 339

Raju, K. P., Srikanth, R., \& Singh, J. 1998, Sol. Phys., 178, 251

Ravindra, B., \& Venkatakrishnan, P. 2003, Sol. Phys., 215, 239

Reeves, E. M. 1976, Sol. Phys., 46, 53

Ruan, P., Wiegelmann, T., Inhester, B., et al. 2008, A\&A, 481, 827

Scherrer, P. H., Bogart, R. S., Bush, R. I., et al. 1995, Sol. Phys., 162, 129

Seehafer, N. 1978, Sol. Phys., 58, 215

Simon, G. W., \& Leighton, R. B. 1964, ApJ, 140, 1120

Schrijver, C. J. 1997, in The Corona and the Solar Wind Near Minimum Activity, ed. A. Wilson, ESA SP-404 (Noordwijk: ESA), 149

Stucki, K., Solanki, S. K., Schühle, U., et al. 2000, A\&A, 363, 1145

Teriaca, L., Banerjee, D., \& Doyle, J. G. 1999, A\&A, 349, 636

Tian, H., Tu, C.-Y., He, J.-S., \& Marsch, E. 2007, Adv. Space Res., 39, 1853

Tian, H., Tu, C.-Y., Marsch, E., He, J.-S., \& Zhou, G.-Q. 2008a, A\&A, 478, 915

Tian, H., Marsch, E., Tu, C.-Y., Xia, L.-D., \& He, J.-S. 2008b, A\&A, 482, 267

Tu, C.-Y., Zhou, C., Marsch, E., et al. 2005a, Science, 308, 519

Tu, C.-Y., Zhou, C., Marsch, E., et al. 2005b, ApJ, 624, L133

Tu, C.-Y., Zhou, C., Marsch, E., et al. 2005c, Proceedings of SW 11-SOHO 16, Connecting Sun and Heliosphere, Whistler, Canada, 12-17 June 2005, ESA SP-592

Wiegelmann, T., \& Neukirch, T. 2002, Sol. Phys., 208, 233

Wiegelmann, T., Xia, L. D., \& Marsch, E. 2005, A\&A, 432, L1

Wilhelm, K., Curdt, W., Marsch, E., et al. 1995, Sol. Phys., 162, 189

Wilhelm, K., Dammasch, I. E., Marsch, E., \& Hassler, D. M. 2000, A\&A, 353, 749

Xia, L. D. 2003, Ph.D. Thesis (Göttingen: Georg-August-Univ.)

Xia, L. D., Marsch, E., \& Curdt, W. 2003, A\&A, 399, L5

Xia, L. D., Marsch, E., \& Wilhelm, K. 2004, A\&A, 424, 1025 